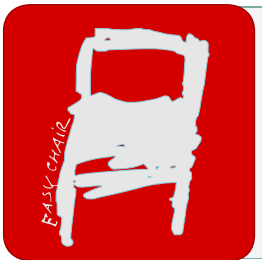

EPiC Series in Health Sciences

Volume 1, 2017, Pages 325-328

CAOS 2017. 17th Annual Meeting of the International

Society for Computer Assisted Orthopaedic Surgery

\title{
Medial Unicompartmental Knee Arthroplasty Using Patient-Specific Instrumentation - Accuracy of Preoperative Planning, Time Saving and Cost Effectiveness
}

\author{
Gesine H. Seeber ${ }^{1 *}$, Kristina Kolbow ${ }^{1}$, Uwe Maus ${ }^{1}$, Alexander Kluge ${ }^{2}$ and \\ Djordje Lazovic ${ }^{1 *}$ \\ ${ }^{1}$ University Hospital for Orthopaedics and Trauma Surgery, Carl von Ossietzky University \\ Oldenburg, Medical Campus Pius-Hospital Oldenburg \\ 2 Department of Interventional and Diagnostic Radiology, Pius-Hospital Oldenburg, Oldenburg \\ gesine.seeber@uol.de, kristina.kolbow@uol.de, uwe.maus@pius- \\ hospital.de, alexander.kluge@pius-hospital.de, djordje.lazovic@pius- \\ hospital.de
}

\begin{abstract}
Since the past few years, patient-specific instrumentation (PSI) has been greatly publicized in knee endoprosthetics. Manufacturers propose advantages such as better accuracy of fit and reduction of both surgical time and operation costs due to preoperative prosthesis planning. Whether these proposed advantages are achieved in medial unicompartmental knee arthroplasty (UKA) remains unclear. This paper presents results from a retrospective analysis of 22 patients (24 knees) who were electively provided with a medial unicompartmental knee arthroplasty using patientspecific instrumentation.
\end{abstract}

\section{Introduction}

Restoration of an optimal alignment is crucial in unicompartmental knee arthroplasty (UKA) since poor implant positioning has been identified as a contributory factor for early prosthesis failure and accelerating cartilage wear in the remaining compartments (Bell et al. 2014). To handle this problem, MRI-based patient-specific instrumentation (PSI) has been greatly publicized in knee endoprosthetics for the past few years. By utilizing PSI the accuracy of the prosthesis components size and alignment should be improved. Besides, shortening of surgical

${ }^{*}$ Created this document

K. Radermacher and F. Rodriguez Y Baena (eds.), CAOS 2017 (EPiC Series in Health Sciences, vol. 1), pp. $325-328$ 
time and reduction of hospital costs are marketed as beneficial (Barrack et al. 2012, Stronach et al. 2014). However, most clinical trials evaluating the use of PSI in total knee arthroplasty surgery could not demonstrate a major benefit - neither functional nor radiological nor economical (Barrack et al. 2012, Stronach et al. 2014, Woolson et al. 2014, Abane et al. 2015). Whether the proposed advantages of PSI are beneficial in medial UKA remains yet unclear. The aim of this study was therefore to evaluate the preoperative planning accuracy, timesavings, and cost effectiveness of PSI application in UKA.

\section{Materials and Methods}

Data from 22 patients (24 knees) presenting with isolated medial unicompartmental knee osteoarthritis were analyzed retrospectively. The study population comprised sixteen men and six women (mean age $61 \pm 8$ years) who were electively provided with a UKA using PSI between June 2012 and October 2014. To evaluate preoperative planning accuracy the following parameters were analyzed: (1) planned vs. implanted femoral component size, (2) planned vs. implanted tibial component size, and (3) planned vs. implanted polyethylene insert size. Since UKA is a less common, technically demanding surgery and depending in a large part on the surgeon's experience (Lobenhoffer 2012), preoperative planning reliability was also evaluated subject to surgeon experience. Another focus of this study lied on the evaluation of the actual surgical time required and the cost effectiveness using PSI.

\section{Results}

Preoperative surgical planning had to be modified intraoperative to a wide extend in order to achieve an optimal outcome. The femoral component had to be adjusted intraoperative in $41.7 \%$ of all cases, the tibial component in $58.3 \%$, and the polyethylene insert in $87.5 \%$. The less experienced surgeon had to change preoperative planning more often compared to the more experienced surgeon. Regardless of experience level, usage of PSI increased surgical time by about seven minutes on average. Overall mean surgical time for a PSI-based UKA was $66.8( \pm 15.2)$ minutes compared to $59.9( \pm 13.3)$ minutes for the same operation using standard instrumentation. A less experienced surgeon needed about nine minutes more for a PSI-based operation (PSI $82.3 \pm 14$ minutes vs. Standard $73 \pm 2.9$ minutes), an experienced surgeon needed about four minutes more for PSI-based operations (PSI 61.7 \pm 12 vs. Standard 58.2 \pm 13.2 minutes). According to the linear regression model, PSI-planning and surgeon inexperience were the main predictors for increased surgical time. Instead of lowering costs, utilizing PSI increased surgical costs by about $1300 \$$ per case. This was due to license fees, enlarged surgical time, and extraordinary expenditure for MRI scans.

\section{Discussion}

Improving the accuracy of fit is one of the primary objectives of PSI. However, in the here presented sample, this primary goal was not achieved. Intraoperative modification rates of $41.2 \%$ to $87.2 \%$ depending on the component have to be judged critically. The more so as other authors report similar values of planning inaccuracy when utilizing PSI (Woolson et al. 
2014, Abane et al. 2015, Bell et al. 2014, Stronach et al. 2013). The preoperative planning inaccuracy of the insert in our study is noticeable. This prosthesis component carries great weight in UKA because it is the goal to not overcorrect the lower limb mechanical axis but rather preserve normal knee kinematics (Lobenhoffer 2012, Panni et al. 2012, Koeck et al. 2011). For that, optimal ligament balancing achieved through the inlay is important (Bell et al. 2014). Although, the inlay is intraoperative the most easily controlled prosthesis component, the utility of PSI is debatable if this important part fits in just $12.5 \%$ of all cases. The exact reason for unsatisfactory preoperative planning accuracy is yet not clear. The surgeon could be a possible error source if not sufficiently familiar with the PSI planning software. Besides, planning based on non-weight bearing MR images is discussed controversially (Paternostre et al. 2014). Those images are of high sensitivity in mapping patient-individual anatomy. However, their geometric precision is subject to confounding factors and deviations can account for up to $0.5 \mathrm{~mm}$ (Rathnayaka et al. 2012). Moreover, drawing inferences about the exact ligamentous state from images recorded in a non-weightbearing condition is difficult. Especially patients with high-order knee arthritis (Kellgren \& Lawrence Grade 3 and 4) exhibit major alignment differences in weightbearing compared to non-weightbearing conditions (Paternostre et al. 2015).

Another goal of PSI is reduction of surgical time. However, our data reveal that even a highly experienced surgeon needed more time for UKA with the use of PSI than with standard instrumentation. Reasons for prolonged surgical time could be the greater preparation effort until exact fitting of PSI templates is achieved. Furthermore, positioning of PSI has to be repeatedly controlled, because even rough mal-positioning is often not visually or haptically recognizable.

A main idea of PSI is to simplify surgical procedures and hence enable less experienced surgeons to perform infrequent and demanding operations in a safe manner (Lachiewitz \& Henderson 2013). However, this aspect must be considered critical since surgeon inexperience was a main predictor for planning error in the present study.

Finally, the proposed cost-reduction benefit could not be witnessed in the current data. This is consistent throughout the literature (Heyse et al. 2014, Barrack et al. 2012, Slover et al. 2012). As long as surgeons cannot rely on their preoperative PSI planning, all trays have to be in place intraoperative in order to have resource to standard instrumentation or different test components. This does not reduce set-up times or instrumental effort.

This study has some limitations. All patients were provided with the same MRI-based PSI UKA model. Hence, no conclusions can be drawn about the accuracy of other PSI models. Moreover, the analyzed sample was rather small. However, indication for medial UKA is still narrow, so that large samples are hard to get. The primary goal for this study was to analyze operational procedures. Hence, pre- and postoperative clinical data are missing.

\section{Conclusion}

The advertised advantages of PSI could not be supported on the basis of the here analyzed data. At this time, the disadvantages of this technology are predominating in terms of additional costs, extended surgical time and insufficient preoperative planning accuracy. Having in mind that up to now no single study was able to demonstrate better outcomes in terms of alignment and/or function by comparing PSI to standard instrumentation, additional data are required before PSI can be recommended for routine use in medial UKA. 


\section{References}

Abane L, Anract P, Boisgard S, et al. A comparison of patient-specific and conventional instrumentation for total knee arthroplasty: a multicentre randomised controlled trial. Bone Joint J. 2015; 97-B(1):56-63.

Barrack RL, Ruh EL, Williams BM, et al. Patient specific cutting blocks are currently of no proven value. J Bone Joint Surg. 2012; 94-Br (11):95-99.

Bell SW, Stoddard J, Bennett C, et al. Accuracy and early outcomes in medial unicompartmental knee arthroplasty performed using patient specific instrumentation. Knee. 2014;21:S33 - S36.

Heyse TJ, Lipman JD, Imhauser CW, et al. Accuracy of Individualized Custom Tibial Cutting Guides in UKA. HSS Journal. 2014; 10(3):260-265.

Koeck FX, Beckmann J, Luring C, et al. Evaluation of implant position and knee alignment after patient-specific unicompartmental knee arthroplasty. Knee. 2011; 18(5):294-299.

Lachiewicz PF, Henderson RA. Patient-specific Instruments for Total Knee Arthroplasty. $J$ Am Acad Orthop Surg. 2013;21:513-518

Lobenhoffer P. Unikondylärer Gelenksersatz. Arthroskopie. 2012; 25(3),195-203.

Panni AS, Vasso M, Cerciello S, Felici A. Unicompartmental knee replacement provides early clinical and functional improvement stabilizing over time. Knee Surg Sports Traumatol Arthrosc. 2012; 20(3):579-585.

Paternostre F, Schwab PE, Thienpont E. The difference between weight-bearing and nonweight-bearing alignment in patient-specific instrumentation planning. Knee Surg Sports Traumatol Arthrosc. 2014; 22(3):674-679.

Rathnayaka K, Momot KI, Noser H, et al. Quantification of the accuracy of MRI generated 3D models of long bones compared to CT generated 3D models. Medical Engineering \& Physics. 2012;34:357-363.

Slover JD, Rubash HE, Malchau H, Bosco JA. Cost-effectiveness analysis of custom total knee cutting blocks. J Arthroplasty. 2012; 27(2):180-185.

Stronach BM, Pelt CE, Erickson JA, et al. Patient-specific instrumentation in total knee arthroplasty provides no improvement in component alignment. J Arthroplasty. 2013;29(9), 1705-1708.

Stronach BM, Pelt CE, Erickson J, Peters CL. Patient-specific total knee arthroplasty required frequent surgeon-directed changes. Clin Orthop Relat Res. 2013; 471(1):169- 174.

Woolson ST, Harris AH, Wagner DW, Giori NJ. Component alignment during total knee arthroplasty with use of standard or custom instrumentation: a randomized clinical trial using computed tomography for postoperative alignment measurement. J Bone Joint Surg. 2014;96Am. (5):366-372. 